\title{
FATORES DETERMINANTES PARA SUSPENSÕES DE CIRURGIAS ELETIVAS EM UM HOSPITAL DO DISTRITO FEDERAL
}

\section{Determinants factors for suspension of elective surgeries in a hospital of the Federal District, Brazil}

\section{Factores determinantes para suspensiones de cirugías electivas en un hospital del distrito federal}

\author{
Jacqueline Ramos de Andrade Antunes Gomes ${ }^{1 *}$, Renata Valero Barbosa Franco², \\ Dalyanne Souza Vieira Diniz Morais ${ }^{3}$, Beatriz Coêlho Barbosa ${ }^{4}$
}

RESUMO: Objetivo: Identificar os principais fatores determinantes para a suspensão de cirurgias eletivas em um hospital público do Distrito Federal e calcular a taxa de suspensão de cirurgias. Método: Trata-se de um estudo quantitativo, retrospectivo, descritivo, realizado no centro cirúrgico de um hospital público do Distrito Federal. Resultado: De janeiro a outubro de 2015 foram programadas 6.926 cirurgias, das quais foram realizadas 4.587 e suspensas 2.339 , totalizando uma taxa de suspensão cirúrgica de 33,8\%. O principal motivo de suspensão foram causas injustificadas, com 30,1\%. Conclusão: Os determinantes para suspensão de cirurgias devem ser rigorosamente controladas e restringidas. Para isso, é essencial a conscientização de todos os envolvidos, com o intuito de diminuir os índices encontrados. Os achados permitem realizar uma análise situacional a respeito da assistência prestada e possibilitam identificar fragilidades, melhorar o desempenho e adequar o processo de trabalho às necessidades do paciente e do setor.

Palavras-chave: Cirurgia. Procedimentos cirúrgicos eletivos. Centro cirúrgico.

ABSTRACT: Objectives: To identify the main determinant factors for suspension of elective surgeries in a public hospital of the Federal District, Brazil, and calculate the surgery suspension rate. Method: This is a quantitative, retrospective, and descriptive study carried out at the surgical center of a public hospital of the Federal District. Result: From January to October of 2015, 6,926 surgeries were scheduled, of which 4,587 were performed and 2,339 suspended, totaling a surgery suspension rate of $33.8 \%$. The main reason for suspension was unjustified causes, with $30.1 \%$. Conclusion: The determinants of surgery suspension should be strictly controlled and restricted. To that end, it is essential to raise awareness among all those involved to reduce the rates found. The findings allowed us to conduct a situational analysis of the care provided, identify weaknesses, improve performance, and adapt the work process to the needs of the patient and the area.

Keywords: Surgery. Elective surgical procedures. Surgical center.

RESUMEN: Objetivo: Identificar los principales factores determinantes para la suspensión de cirugías electivas en un hospital público del Distrito Federal y calcular la tasa de suspensión de cirugías. Método: Se trata de un estudio cuantitativo, retrospectivo, descriptivo, realizado en el centro quirúrgico de un hospital público del Distrito Federal. Resultado: De enero a octubre de 2015 fueron programadas 6.926 cirugías, de las cuales fueron realizadas 4.587 y suspendidas 2.339, totalizando una tasa de suspensión quirúrgica del 33,8\%. El principal motivo de suspensión, fueron causas injustificadas, con el 30,1\%. Conclusión: Las determinantes para suspensión de cirugías deben ser rigurosamente controladas y restringidas. Para eso, es esencial la concientización de todos los involucrados, con la idea de disminuir los índices encontrados. Los hallazgos permiten realizar un análisis situacional al respecto de la asistencia prestada y posibilitan identificar fragilidades, mejorar el desempeño y adecuar el proceso de trabajo a las necesidades del paciente y del sector. Palabras-clave: Cirugía. Procedimientos quirúrgicos electivos. Centro quirúrgico.

'Enfermeira; doutora em Ciências da Saúde pela Universidade de Brasília; coordenadora do Programa de Residência de Enfermagem em Centro Cirúrgico em Rede da Secretaria de Estado de Saúde do Distrito Federal - Brasília (DF), Brasil.

2Enfermeira especialista em Enfermagem do Trabalho; enfermeira do trabalho pela Universidade de Brasília - Brasília (DF), Brasil.

${ }^{3}$ Enfermeira especialista em Enfermagem do Trabalho; enfermeira pelo Instituto Federal - Brasília (DF), Brasil.

«Enfermeira; mestranda em Saúde Coletiva pela Universidade de Brasília; professora do Centro Universitário do Instituto de Educação Superior de Brasília - Brasília (DF), Brasil.

*Autor correspondente: jraagdoutoradofsunb@gmail.com

Recebido: 28/02/2018. Aprovado: 21/05/2018.

DOI: 10.5327/Z1414-4425201800040003 


\section{INTRODUÇÃO}

O ato cirúrgico proporciona parâmetros de avaliação de qualidade da assistência em centro cirúrgico (CC) das instituições hospitalares e interfere diretamente no indicador de produtividade. Como critério de avaliação de eficiência, utiliza-se a taxa de suspensão como um indicador de qualidade ${ }^{1}$. A taxa de suspensão de cirurgia é calculada por meio do número de cirurgias suspensas dividido pelo total de cirurgias programadas, multiplicado por 100, de acordo com o Ministério da Saúde (MS)2.

Qualquer procedimento programado com antecedência é caracterizado como procedimento eletivo. Nesse caso, há mais tempo para o paciente e a equipe se prepararem para a intervenção, reduzindo ao máximo o risco de intercorrências ${ }^{3}$.

A cirurgia é um processo terapêutico que possui várias finalidades e desencadeia inúmeras reações fisiológicas e psicológicas no paciente, bem como o coloca em risco de morte e perda de órgãos, podendo gerar incapacidades e transformar completamente a vida do indivíduo submetido e das pessoas que o cercam ${ }^{3,4}$.

O bem-estar do indivíduo em situação cirúrgica deve ser o principal objetivo da equipe de saúde 5 . No momento em que o paciente se prepara para o procedimento cirúrgico surgem, automaticamente, muitas preocupações e expectativas sobre a intervenção que será realizada, e quando cancelado, pode gerar sentimento de frustração $o^{1,6}$.

Os inúmeros transtornos causados devido à suspensão de uma cirurgia podem ser analisados por duas vertentes: uma com base nas repercussões geradas para o indivíduo e seus familiares e outra avaliando as consequências causadas para a instituição de saúde e os profissionais ali atuantes ${ }^{7}$.

Alguns dos principais motivos para suspensão dos procedimentos cirúrgicos na literatura são: questões administrativas e estruturais; condições clínicas inadequadas; atrasos da equipe cirúrgica; falhas de comunicação entre equipe médica, CC e unidades de internação; falta de insumos necessários; falhas no pré-operatório e falta de exames previamente solicitados ${ }^{1,8}$.

Os prejuízos para a instituição são inúmeros e vão desde o preparo de um material específico e da sala cirúrgica até os recursos humanos mobilizados ${ }^{1,9}$. Todos esses fatores acarretam prejuízo financeiro, causado pela deficiência do processo, pois além dos fatores supracitados, ainda há o aumento da taxa de permanência do paciente, diminuição da rotatividade do hospital e aumento do risco de infecção relacionado a assistência à saúde ou outras complicações ${ }^{10}$.

Estima-se que cerca de $60 \%$ dos cancelamentos cirúrgicos eletivos sejam potencialmente evitáveis ${ }^{1}$. A realização de uma assistência multidisciplinar planejada e articulada, a elaboração de projetos com gestão eficiente e avaliação constante da qualidade e da produtividade, assim como do impacto gerado a partir das ações sugeridas, são de extrema importância para o bom funcionamento de uma unidade de CC, além de tornar mais eficiente a detecção de condutas e ações que necessitem ser revisadas, impedindo, assim, o acúmulo de fragilidades a serem resolvidas ${ }^{1,4}$.

Os procedimentos cirúrgicos são atividades que demandam preparo complexo e que, ao serem suspensos, causam impacto físico e emocional aos pacientes, além de prejuízo organizacional e custo financeiro elevado para as instituições. Nesse sentido, é necessário entender com maiores detalhes as repercussões geradas a partir de uma análise de variáveis implicadas na prática cirúrgica, com vistas à minimização de danos aos pacientes e ao uso racional de recursos materiais, financeiros e humanos para essa atividade.

\section{OBJETIVOS}

- Calcular a taxa de suspensão de cirurgias eletivas em um hospital público do Distrito Federal;

- Identificar os fatores determinantes da suspensão de cirurgias eletivas em um hospital público do Distrito Federal.

\section{MÉTODO}

Trata-se de uma pesquisa descritiva, com abordagem quantitativa, retrospectiva, realizada no CC de um hospital público do Distrito Federal. O estudo quantitativo visa observar, registrar e descrever as características de uma amostra ${ }^{11}$.

A amostra foi composta por dados de 6.926 cirurgias eletivas, contempladas no arquivo de mapas cirúrgicos da instituição, entre os meses de janeiro e outubro de 2015. Como critérios de inclusão foram consideradas as cirurgias eletivas suspensas no período de janeiro a outubro de 2015 e que na justificativa de suspensão estivesse escrito "sem motivo". Excluíram-se os dados que não estavam claros e/ ou corretamente preenchidos. As cirurgias de urgência e emergência não foram inseridas no estudo, pois a rotatividade de pacientes é frequente.

O instrumento utilizado para a coleta dos dados foi construído pelos pesquisadores para facilitar a organização e análise dos dados. A partir da justificativa da suspensão cirúrgica, os dados foram contabilizados no formulário e divididos em tópicos de enquadramento de determinantes: assistenciais, administrativos, estruturais, ausência do paciente, condições clínicas insatisfatórias, causas injustificadas, procedimento não autorizado e outros. 
No que diz respeito aos motivos das suspensões, foram consideradas injustificadas as cirurgias que apresentavam a descrição "sem motivo" ou as que foram substituídas. No motivo "outros", incluíram-se aquelas que tiveram avanço de horário, erro no agendamento ou desistência do paciente. Os motivos administrativos foram aqueles nos quais constavam falta de prontuários, ausência de funcionários, procedimento de urgência em outro paciente no mesmo horário, deficiência de materiais ou equipamentos, cirurgias realizadas anteriormente e pacientes sem indicação cirúrgica. Na ausência do paciente, foram considerados os que não compareceram para a realização do procedimento.

Como motivos estruturais, encaixaram-se a falta de leitos (incluindo as unidades de tratamento intensivo), falta de sala operatória e falha nos serviços de manutenção no CC. Nas condições clínicas insatisfatórias, cabem os pacientes debilitados no pré-operatório ou que sofreram alteração de conduta médica. Dentre os motivos assistenciais, encontramos inadequação do preparo pré-operatório (ausência de jejum, administração de medicações contraindicadas no pré-operatório, ausência de reserva de sangue e hemoderivados, não realização de avaliação pré-anestésica e exames complementares incompletos). E as não autorizadas foram aquelas nas quais não houve a internação do paciente, devido à falta de autorização da guia, do laudo de internação ou do cartão do Sistema Único de Saúde (SUS).

Os mapas cirúrgicos foram selecionados no arquivo do hospital, por meio do instrumento de coleta. Os dados foram contabilizados e posteriormente analisados e comparados por meio da frequência das variáveis pesquisadas, com auxílio do software SPSS 22.0. As taxas de suspensão cirúrgica foram calculadas dividindo o número de cirurgias suspensas pelo total de cirurgias eletivas, multiplicado por $100^{2}$.

Em relação aos aspectos éticos, o projeto foi submetido à apreciação do Comitê de Ética em Pesquisa (CEP) da Fundação de Ensino e Pesquisa (FEPECS), da Secretaria de Estado de Saúde do Distrito Federal, e aprovado sob o número CAAE 52294315.4.0000.5553, conforme preconiza a Resolução $\mathrm{n}^{\mathrm{o}} 466 / 2012$, do Conselho Nacional de Saúde, que aborda as diretrizes para pesquisas envolvendo seres humanos ${ }^{12}$.

\section{RESULTADOS}

No período estudado (entre janeiro e outubro de 2015) foram programadas 6.926 cirurgias, dessas, foram realizadas 4.587 e suspensas 2.339 , totalizando uma taxa de suspensão cirúrgica de $33,8 \%$. Das cirurgias programadas, $610(8,8 \%)$ foram substituídas, sem descrição do motivo; sendo assim, foram agrupadas dentro do motivo de cirurgias suspensas "injustificadas".

O maior quantitativo de cirurgias suspensas foi nos meses de junho, julho e agosto, somando o percentual de $38,5 \%$, conforme a Figura 1.

No mapa cirúrgico foram encontradas todas as cirurgias suspensas, apresentando os motivos correspondentes. Verificou-se que das 584 cirurgias suspensas classificadas como motivo "outros", 100,0\% dos casos foram ocasionados pelo avanço de horário da cirurgia anterior. A partir disso, foram enquadradas nos grupos de determinantes ordenados pelo estudo, de acordo com a Tabela 1.

\section{DISCUSSÃO}

O hospital pesquisado apresentou taxa de suspensão de $33,8 \%$, sendo considerado superior aos encontrados na literatura,

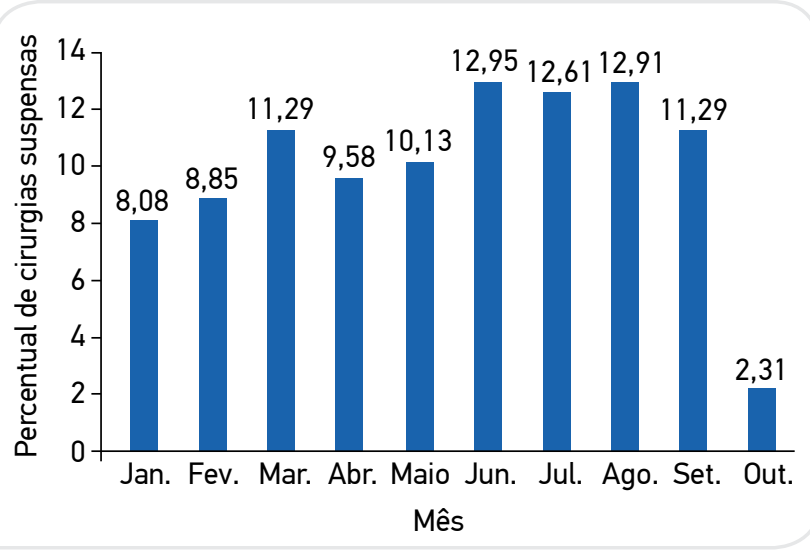

Figura 1. Percentual de cirurgias suspensas de janeiro a outubro de 2015.

Tabela 1. Quantitativo de cirurgias suspensas de acordo com o motivo.

\begin{tabular}{|l|c|c|}
\hline Motivo & $\begin{array}{c}\text { Quantitativo absoluto } \\
\text { de cirurgia suspensa }\end{array}$ & Percentual \\
\hline Injustificadas & 703 & 30,1 \\
\hline Outros & 584 & 25,0 \\
\hline Administrativo & 510 & 21,8 \\
\hline Ausência de paciente & 249 & 10,6 \\
\hline Estruturais & 157 & 6,7 \\
\hline Condições clínicas & 82 & 3,5 \\
\hline insatisfatórias & 51 & 2,2 \\
\hline Assistenciais & 02 & 0,1 \\
\hline Não autorizado & &
\end{tabular}


que variam entre 14,14 e $27,4 \%{ }^{1,5,8,9}$. O alto índice pode ser reflexo da falta de planejamento das ações que envolvem todo o processo da programação cirúrgica. Segundo a literatura, essas altas taxas são comuns em hospitais públicos", constituindo uma avaliação negativa para a instituição, uma vez que é referência na unidade da federação.

Durante o período da pesquisa, 610 cirurgias foram substituídas, sem descrição do motivo no mapa cirúrgico; sendo assim, o procedimento que não ocorreu foi considerado suspenso e injustificado. Esse fato culminou em uma alta porcentagem, fazendo com que as cirurgias injustificadas ficassem em primeiro lugar com 703 procedimentos suspensos, representando $30,1 \%$ das causas.

Tratando-se de cirurgias eletivas, a não realização do procedimento pode ocorrer por falha nas visitas pré-operatórias, construção inadequada do mapa cirúrgico, como tempo de antecedência de sua construção, falta de programação por parte do cirurgião, agendamento de paciente fictício para assegurar sala e erro no agendamento cirúrgico. Todos esses fatores são de possível resolução e impactariam diretamente na redução das taxas de suspensão cirúrgica, assim como o preenchimento correto dos motivos de substituição traria mais fidedignidade aos dados. Esse achado mostra a necessidade de conscientização dos profissionais, pois apenas a partir do conhecimento da causa é que se consegue propor medidas para correção da fragilidade ${ }^{1}$.

Os meses em que mais se encontraram suspensões cirúrgicas foram junho, julho e agosto, que juntos somam $38,4 \%$ das suspensões. No que diz respeito aos motivos determinantes para a suspensão de cirurgias, temos como principal fator as cirurgias suspensas injustificadas $(30,1 \%)$, seguidas de outros motivos $(25,0 \%)$.

O resultado diverge dos dados encontrados em outras pesquisas, que citam entre os principais motivos as condições clínicas insatisfatórias do paciente e os motivos assistenciais, por falta de exames ${ }^{1,5,9}$. No presente estudo, esses dois motivos ficaram em sexto e sétimo lugares, com 3,5 e 2,2\%, respectivamente, podendo ter como causa a falta de dados que pudessem caracterizar os reais motivos da suspensão cirúrgica ou devido ao déficit de profissionais na instituição.

Quanto aos motivos administrativos, o resultado foi significativo, totalizando $21,8 \%$, divergindo do estudo realizado em um hospital de São Paulo, em que os motivos administrativos representaram apenas $13,9 \%{ }^{6}$. Tal causa pode estar relacionada com o dimensionamento incorreto dos profissionais e insumos escassos na instituição.

Os aspectos estruturais representam $6,7 \%$ das cirurgias suspensas; a falta de leito foi a justificativa mais identificada nesse grupo, corroborando a literatura ${ }^{7}$. Esse fator pode estar relacionado a falhas no planejamento das cirurgias e pode ser solucionado por meio da confirmação dos leitos no dia anterior à cirurgia.

Em relação ao quantitativo total de suspensão cirúrgica, observou-se uma média de 234 cirurgias canceladas por mês. Propõe-se que, para minimizar esse índice, seja feita uma análise situacional mensal por parte dos gerentes do CC, com o intuito de detectar as possíveis causas que impedem que o fluxo correto aconteça e traçar ações cabíveis para a resolução dos problemas encontrados.

Em relação aos determinantes de suspensões cirúrgicas mensais, em fevereiro, abril, maio, junho e julho, o principal motivo foi o "injustificado". Em janeiro, março e agosto, foi "outros" motivos, e em setembro e outubro, os problemas administrativos obtiveram destaque.

Podemos justificar o grande número de problemas administrativos nos meses de setembro e outubro devido à greve de funcionários da saúde, que ocorreu no período e gerou grande impacto no funcionamento do CC. Somente as cirurgias de urgência estavam sendo realizadas, causando a suspensão das cirurgias eletivas por falta de recursos humanos.

A literatura mostra que $60 \%$ dos fatores determinantes para as suspensões de cirurgias poderiam ser previstos e evitados. Para tanto, cabe aos gerentes do CC redefinir modelos, estipular metas, estudar as peculiaridades do setor e agrupar fragilidades e potencialidades, para, assim, rever o planejamento do mapa cirúrgico, a visita pré-operatória, o gerenciamento de materiais e os recursos humanos ${ }^{5,6}$. Obter o controle adequado de todos esses fatores proporciona maior segurança para o paciente, diminui o tempo desnecessário de internação, reduz significativamente os custos e otimiza a produtividade do setor ${ }^{4}$.

A equipe de saúde, com destaque à equipe de enfermagem por possuir papel fundamental no preparo e manejo do paciente em situação pré-operatória, é de grande relevância para o tema em estudo, pois são os profissionais atuantes no sistema e responsáveis pela dinâmica da instituição. Esses profissionais necessitam obter conhecimento acerca da situação vivenciada e das problemáticas existentes em seu ambiente de trabalho para que a atuação seja mais efetiva. Esta pesquisa permite o embasamento para o conhecimento e possível planejamento e execução de uma melhor organização da assistência, resultando em melhoria para o paciente, assim como para a instituição.

As limitações da pesquisa foram a falta de manuscritos legíveis e a incompletude dos mapas cirúrgicos. Observa-se que é pertinente a realização de um trabalho acerca da operacionalização do CC, a fim de possibilitar à instituição uma análise mais precisa a respeito da produtividade e do 
desempenho do setor. Uma pesquisa sobre análise de custos por cirurgias suspensas e realizadas favoreceria a reflexão da equipe e dos gerentes do CC.

\section{CONCLUSÃO}

A pesquisa permitiu realizar uma análise situacional a respeito da assistência prestada, possibilitando melhorar o desempenho $\mathrm{e}$ adequar o processo de trabalho às necessidades do paciente $\mathrm{e}$ do CC. O intuito é orientar e contribuir com a gerência do setor e os profissionais que ali trabalham, a partir dos resultados obtidos, para que os transtornos gerados pela suspensão de cirurgias aos pacientes, aos familiares e à instituição sejam minimizados.
Os fatores determinantes para a ocorrência de suspensão cirúrgica devem ser rigorosamente controlados e restringidos. A taxa geral de suspensão encontrada no estudo foi de $33,8 \%$ e, no que diz respeito aos motivos determinantes para tal, encontraram-se como principal fator as causas injustificadas com representatividade de $30,1 \%$, seguidas de outros motivos $(25,0 \%)$, motivos administrativos $(21,8 \%)$, ausência do paciente $(10,6 \%)$ e motivos estruturais $(6,7 \%)$.

Por fim, cabe reforçar a necessidade da conscientização dos profissionais envolvidos, como a equipe médica e os profissionais de enfermagem, para o preenchimento completo e correto dos dados das cirurgias suspensas no sistema informatizado e capacitação para o correto preenchimento, possibilitando planejar as ações com maior resolutividade.

\section{REFERÊNCIAS}

1. Carvalho TA, Sobral CB, Marinho PML, Rodriguez EOL, Campos MPA. Suspensão de cirurgias em um hospital universitário. Rev SOBECC. 2016;21(4):186-91. https://doi.org/10.5327/Z1414-4425201600040002

2. Moraes PGS, Pacheco NMD, Silva RGS, Silva PCV. Clinical and organizational factors related to cancellation of surgical procedures. J Nurs UFPE [Internet]. 2017 [citado 26 nov. 2017];1 1 (7):2645-53. Disponível em: https://doi.org/10.5205/1981-8963-v1 1i7a23436p2645-2653-2017

3. Carvalho RWF, Pereira CU, Laureano Filho JR, Vasconcelos BCE. 0 paciente cirúrgico: parte I. Rev Cir Traumatol Buco-maxilo-fac [Internet]. 2010 [citado 30 nov. 2017];10(4):85-92. Disponível em: http://www.revistacirurgiabmf.com/2010/V10n4/12.pdf

4. Cihoda JH, Alves JR, Fernandes LA, Souza Neto EP. Analysis for the causes of surgical cancellations in a Brazilian university hospital. Care Management J [Internet]. 2015 [citado 29 nov. 2017];16(1):417. Disponível em: https://doi.org/10.1891/1521-0987.16.1.41

5. Sampaio CEP, Gonçalves RA, Seabra Júnior HC. Determination of surgery suspension factors and their constributions with nursing assistance. Care Online [Internet]. 2016 [citado 30 nov. 2017]:8(3):481320. Disponível em: http://dx.doi.org/10.9789/2175-5361.2016. v8i3.4813-4820

6. Moreira LR, Xavier APR, Moreira FN, Souza LCM, Araújo OC, Santos TMB, et al. Avaliação dos motivos de cancelamento de cirurgias eletivas. Enfermagem ver [Internet]. 2016 [citado 27 nov. 2017];19(2). Disponível em: http://periodicos.pucminas.br/index.php/enfermagemrevista/ article/view/13156/10301

7. Hori Y, Nakayama A, Sakamoto A. Surgery cancellations after entering the operating room. JA Clinical Reports [Internet]. 2016 [citado 27 nov. 2017];2(40). Disponivel em: https://doi.org/10.1186/s40981-016-0066-1

8. Callegari DC, Batista HMT, Silveira GBM, Bentes IMS, Rocha RPB, Sampaio $L A$, et al. Monitoring the suspension of surgical procedures. Int Arch Med [Internet]. 2015 [citado 30 nov 2017]:8. Disponível em: http://dx.doi.org/10.3823/1710

9. Macedo JM, Kano JA, Braga EM, Garcia MA, Caldeira SM. Cancelamento de cirurgias em um hospital universitário: causas e tempo de espera para novo procedimento. Rev SOBECC. 2013;18(1):26-34.

10. Botazini NO, Carvalho R. Cancellation of surgeries: an integrative literature review. Rev SOBECC. 2017;22(4):230-44. http://dx.doi. org/10.5327/Z1414-4425201700040008

11. Fontelles MJ, Simões MG, FariasSH, Fontelles RGS. Metodologia dapesquisa cientifica: diretrizes para a elaboração de um protocolo de pesquisa. Rev Eletron Educação [Internet]. 2009 [citado 28 nov. 2017]:6(1). Disponível em: https://cienciassaude.medicina.ufg.br/up/150/o/Anexo_C8_NONAME.pdf

12. Brasil. Conselho Nacional de Saúde (CNS). Resolução n 466, de 12 de dezembro de 2012. Aprova as diretrizes e normas regulamentadoras de pesquisas envolvendo seres humanos. Diário Oficial da União. 13 jun. 2013. 